International Journal of Bifurcation and Chaos, Vol. 7, No. 8 (1997) 1775-1790

(C) World Scientific Publishing Company

\title{
PULSES, FRONTS AND CHAOTIC WAVE TRAINS IN A ONE-DIMENSIONAL CHUA'S LATTICE
}

\author{
V. B. KAZANTSEV, V. I. NEKORKIN \\ The Nizhny Novgorod State University \\ 23 Gagarin Ave., 603600 Nizhny Novgorod, Russia \\ M. G. VELARDE \\ Instituto Pluridisciplinar, Universidad Complutense, \\ Paseo Juan XXIII, No 1, Madrid 28 040, Spain
}

Received March 2, 1997; Revised September 20, 1997

\begin{abstract}
We show how wave motions propagate in a nonequilibrium discrete medium modeled by a onedimensional array of diffusively coupled Chua's circuits. The problem of the existence of the stationary wave solutions is reduced to the analysis of bounded trajectories of a fourth-order system of nonlinear ODEs. Then, we study the homoclinic and heteroclinic bifurcations of the ODEs system. The lattice can sustain the propagation of solitary pulses, wave fronts and complex wave trains with periodic or chaotic profile.
\end{abstract}

\section{Introduction}

Many systems modeling processes in nonequilibrium excitable media are known to display solutions localized in space and steadily translating. Solitary pulses, fronts and wave trains propagating with a constant velocity are particular cases. Examples come from all areas of science and engineering. Take, for instance, waves in fluids [Nepomnyashchy \& Velarde, 1994], concentration waves in oscillatory reaction-diffusion systems [Zhabotinsky, 1974; Kuramoto, 1984], waves in optical fibers [Hasegawa \& Kodama, 1995; Huang \& Velarde, 1996], pulses and pulse trains in long arrays of Josephson junctions [Lonngren \& Scott, 1995], transmission of excitation in neural fibers [Murray, 1993], etc. Models allowing to describe such processes are appropriate Ginzburg-Landau equations [Van Saarloos \& Hohenberg, 1992], the Korteweg de Vries equation [Nekorkin \& Velarde, 1994; Velarde et al., 1995; Christov \& Velarde, 1995], the model of Fitz-Hugh Nagumo [Murray, 1993] and their generalizations [Nepomnyashchy \& Velarde, 1994].

There has also been growing interest in models composed of coupled nonlinear oscillators located at the junctions or sites of a space lattice. Such systems are also appropriate models for continuous media describing well, for example, the phenomena of pattern formation [Nekorkin \& Chua, 1993], wave propagation [Perez-Munuzuri et al., 1993; Nekorkin et al., 1995, 1996] and spatially chaotic or spatiotemporal chaotic processes [Ogorzalek, 1995; Nekorkin et al., 1995]. On the other hand, lattice models naturally arise when using arrays of Josephson junctions, arrays of reaction cells, neural networks, arrays of electronic oscillators, etc., whose dynamical behavior can be quite complex both in time and in space [Perez-Munuzuri et al., 1993; Winfree, 1991; Nekorkin et al., 1995; Perez Marino et al., 1995]. Such lattices correspond to discrete nonequilibrium media. Finally, such models can be used suitably for simulation of a given natural process by means of, for example, appropriate electronic circuits which would exhibit a required collective behavior. This is of great importance both from the applied viewpoint and for the understanding of dynamical processes in nature.

We study the dynamics of a one-dimensional array of diffusively coupled Chua's circuits [Chua, 
1993; Madan, 1993]. In recent studies such array has been used for modeling biological fibers, neural networks, reaction-diffusion systems, etc. It has been found that the array can exhibit pattern formation or spatial disorder, propagation of wave fronts, reentry initiation of pulses in two coupled arrays, spiral and scroll waves in two-dimensional arrays, etc. The possibility of traveling pulses and wave trains has been shown in [Nekorkin et al., 1995] for the case of inductive coupling between cells. However, generally these solutions although may be long lasting structures they are not stable. In this paper we discuss this problem for the case of resistive, diffusive coupling and show how the array can be considered as an excitable "fiber" capable of sustaining the propagation of various types of stable travelling waves including single pulses or fronts and complex wave trains with a periodic or a chaotic sequence of pulses. The profiles of possible travelling waves are derived as bounded trajectories of the fourth-order system of ODEs underlying the original space-dependent problem.

\section{Model}

The dynamics of 1-D lattice of diffusively coupled Chua's circuits can be described in dimensionless form by the following set of ODEs (see e.g. [Nekorkin \& Chua, 1993]):

$$
\left\{\begin{array}{l}
\dot{x}_{j}=\alpha\left(y_{j}-x_{j}-f\left(x_{j}\right)\right)+d\left(x_{j-1}-2 x_{j}+x_{j+1}\right) \\
\dot{y}_{j}=x_{j}-y_{j}+z_{j} \\
\dot{z}_{j}=-\beta y_{j}-\gamma z_{j} \\
\qquad j=1,2, \ldots, N
\end{array}\right.
$$

where $f(x)$ describes the symmetric three-segment piecewise-linear function

$$
f(x)= \begin{cases}b x-a-b & \text { if } x \geq 1 \\ -a x & \text { if }-1<x<1 \\ b x+a+b & \text { if } x \leq-1\end{cases}
$$

with $a>0$ and $b>0$. The other parameters of the system $\alpha, \beta, \gamma, d$ are also taken positive. We shall consider two types of boundary conditions: (i) zero-flux conditions $x_{0}=x_{1}, x_{N+1}=x_{N}$, and (ii) periodic conditions $x_{0}=x_{N}, x_{N+1}=x_{1}$. The latter describes a circular array.

The system (1) has three equilibria or fixed points corresponding to the homogeneous steady states of the array. These states are

$$
\begin{aligned}
O & :\left\{x_{j}=y_{j}=z_{j}=0\right\}, \\
P^{+} & :\left\{x_{j}=x_{0}, y_{j}=y_{0}, z_{j}=z_{0}\right\}, \\
P^{-} & :\left\{x_{j}=-x_{0}, y_{j}=-y_{0}, z_{j}=-z_{0}\right\} .
\end{aligned}
$$

where

$$
\begin{aligned}
& x_{0}=\frac{(b+a)(\gamma+\beta)}{(\gamma b+\beta(b+1))}, \\
& y_{0}=\frac{(b+a) \gamma}{(\gamma b+\beta(b+1))}, \\
& z_{0}=-\frac{(b+a) \beta}{(\gamma b+\beta(b+1))} .
\end{aligned}
$$

It has been already shown in [Nekorkin et al., 1993] that for each set of the parameter values the "outer" states $P^{-}$and $P^{+}$are locally asymptotically stable while the trivial state $O$ is unstable. Thus the array is a discrete medium with two excitable steady states. We now show that this medium is able to sustain stable localized solutions (fronts, pulses, pulse trains) travelling in space with definite velocity.

\section{Possible Profiles of Travelling Waves}

Here we prove the existence of travelling wave solutions in the system (1) and determine some characteristics of the waves (profile form, velocity of propagation).

\subsection{Travelling waves}

Let us look for a solution of the system (1) in the form of a travelling wave. We pose

$$
\begin{aligned}
& x_{j}(t)=x(\xi) \\
& y_{j}(t)=y(\xi) \\
& z_{j}(t)=z(\xi),
\end{aligned}
$$

where $\xi=t+j h$ is a coordinate moving along the array with a constant velocity $c=1 / h$. Thus, $(x(\xi), y(\xi), z(\xi))$ describes a space profile steadily translating with the velocity $c$. For solutions of the form (3) the system (1) is reduced to

$$
\left\{\begin{aligned}
\dot{x}= & \alpha(y-x-f(x))+d(x(\xi-h) \\
& -2 x(\xi)+x(\xi+h)) \\
\dot{y}= & x-y+z \\
\dot{z}= & -\beta y-\gamma z
\end{aligned}\right.
$$


where the dot denotes the differentiation with respect to the moving coordinate $\xi$. In the continuum approximation (see e.g. [Nekorkin et al., 1995]), i.e. when the spatial grid of the solution profile is significantly finer than the spatial grid of the discrete array, it is possible to change the difference term in Eq. (4) by using the second derivative $\ddot{x}$. Then, after simple transformations we obtain for traveling waves the following fourth-order system of ODEs:

$$
\left\{\begin{array}{l}
\dot{x}=u \\
k \dot{u}=u-\alpha(y-x-f(x)) \\
\dot{y}=x-y+z \\
\dot{z}=-\beta y-\gamma z
\end{array}\right.
$$

where $k=d / c^{2}$ is a parameter characterizing the dependence of the velocity of the waves on the magnitude of the diffusion $d$.

Any bounded trajectory of the system (4) determines the possible profile of a traveling wave which steadily translates along the array with a constant velocity. The trivial translating solutions homogeneous steady states $O, P^{ \pm}$of the array correspond to the steady points of the system (4) which we denote by the same letters $O, P^{ \pm}$, respectively. Nontrivial solutions homoclinic to the points $P^{ \pm}$define solitary pulses propagating with respect to the "background" steady states $P^{ \pm}$, respectively. Orbits which asymptotically tend with $\xi \rightarrow \pm \infty$ to the different steady points (heteroclinic orbits) correspond to the traveling fronts selecting the terminal homogeneous stable state of the arrays. The system (4) admits various homoclinic and heteroclinic solutions and, besides, solutions of extremely complex profile, as we see further below.

\subsection{Phase space analysis}

Let us analyze the properties of the trajectories of the system (4) before embarking in the study of homoclinic and heteroclinic bifurcations.

Note, first, that by the symmetry properties of the function $f(x)$, the vector field of the system (4), is invariant under space reflection

$$
(x, u, y, z) \rightarrow(-x,-u,-y,-z) .
$$

Hence, for any given trajectory of the system (4) another trajectory defined by this transformation coexists in the phase space. For instance, if there appears a homoclinic orbit of the steady point $P^{+}$, the orbit homoclinic to the point $P^{-}$ also appears, and its profile is defined by the space reflection (6).

Due to the piecewise-linearity of the function $f(x)$, the four-dimensional phase space of the system (4) can be divided in three regions. In each of these regions, motions are governed by linear systems. The planes making this division are

$$
U^{+}:\{x=1\} \quad \text { and } \quad U^{-}:\{x=-1\} .
$$

In each linear region the dynamics of the system (4) is defined only by four eigenvalues of the linear matrix and their corresponding eigenvectors which define the manifolds of the steady points $O, P^{ \pm}$.

Let the parameters of the array unit be $\{a=$ $1.5, b=2, \beta=0.5, \gamma=0.01\}$ and take $\{\alpha, k\}$ as the control parameters. For each $\{\alpha>0, k>0\}$ it can be shown that the eigenvalues corresponding to the "outer" points $P^{ \pm}$are

$$
\left\{\lambda_{1}>0, \lambda_{2}<0, \lambda_{3,4}=-h+i \omega(h>0)\right\} .
$$

Then, the points $P^{ \pm}$have a one-dimensional unstable manifold $W_{u}\left(P^{ \pm}\right)$and a three-dimensional stable manifold $W_{s}\left(P^{ \pm}\right){ }^{1}$ Within the corresponding linear regions the $W_{s}\left(P^{ \pm}\right)$represents the separatrix plane in the four-dimensional phase space and $W_{u}\left(P^{ \pm}\right)$the line containing point $P^{ \pm}$and two halflines $W_{1}$ and $W_{2}$ extending to the different sides of separatrix plane. Figure 1 illustrates qualitatively the arrangement of the manifolds $W_{s}\left(P^{+}\right)$ and $W_{u}\left(P^{+}\right)$relative to the division plane $U^{+}$in the phase space of the system (4). Let us denote by $M_{u}^{+}$the intersection point of the half-line $W_{1}$ and the plane $U^{+}$, and by $l_{s}^{+}$the line of intersection of the separatrix plane $W_{s}\left(P^{+}\right)$and the plane $U^{+}$. By the symmetry property (6) of the system (4) the point $M_{u}^{-}$and the line $l_{s}^{-}$with analogous properties are well defined in the plane $U^{-}$

$$
\begin{gathered}
M_{u}^{ \pm}:\left\{W_{u}\left(P^{ \pm}\right) \cap U^{ \pm}\right\}, \\
l_{s}^{ \pm}:\left\{W_{s}\left(P^{ \pm}\right) \cap U^{ \pm}\right\} .
\end{gathered}
$$

The line $l_{s}^{ \pm}$divides its neighborhood in the plane $U^{ \pm}$in two regions which we denote by $D_{\infty}^{ \pm}$and $D_{n}^{ \pm}$as shown in Figs. 1(a) and 1(b). The trajectories intersecting $U^{ \pm}$within $D_{\infty}^{ \pm}$travel "above"

\footnotetext{
${ }^{1}$ The steady point $O$ depends on control parameters. It can be either asymptotically stable (all eigenvalues have negative real part) or saddle (saddle-focus) with two eigenvalues having positive real parts.
} 


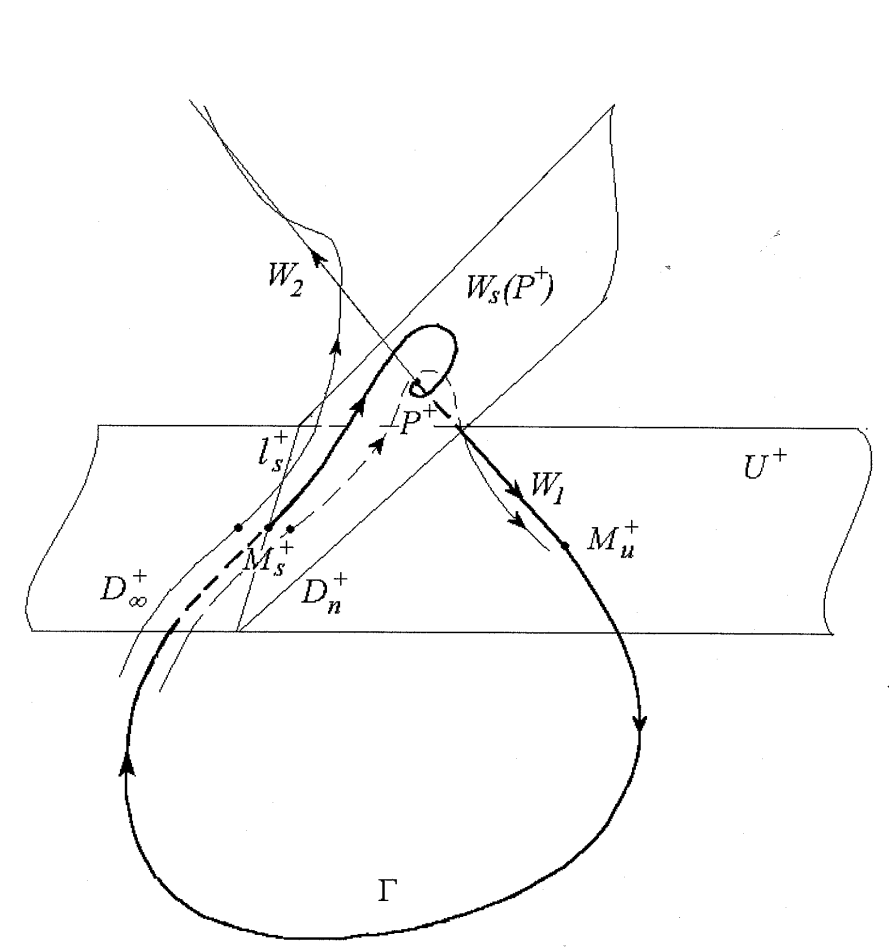

(a)

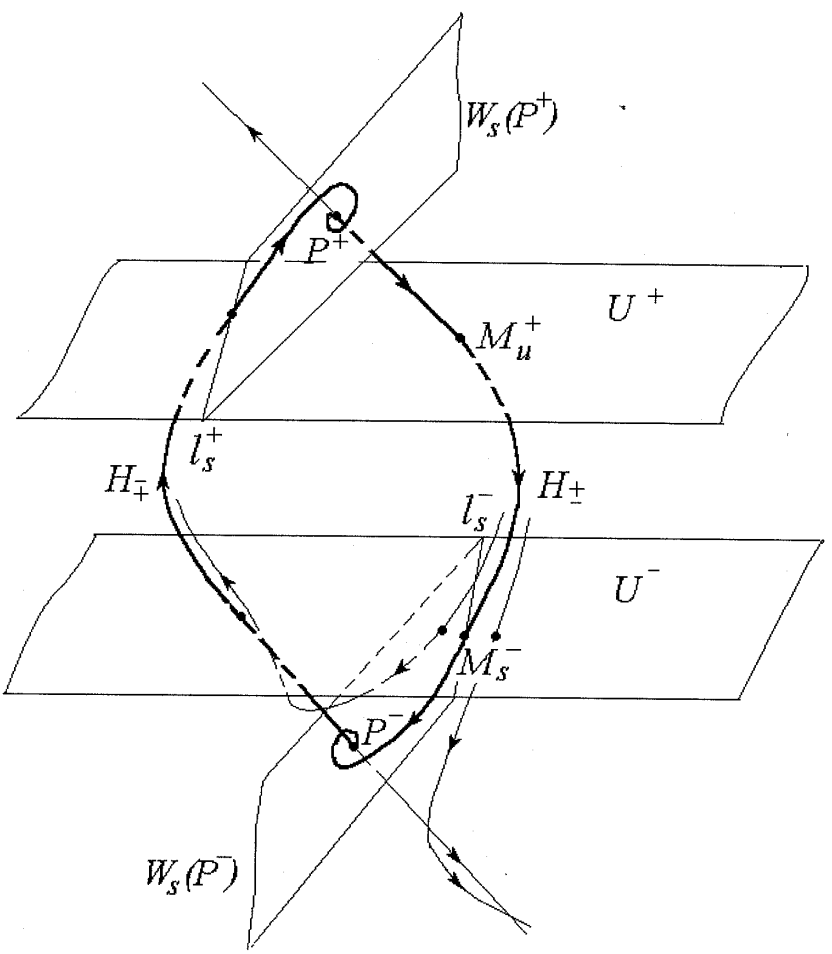

(b)

Fig. 1. Manifolds of the steady point $P^{ \pm}$in the phase space of the system (4). (a) Qualitative behavior of the trajectory forming a homoclinic loop and neighboring trajectories. If the point $M_{u}^{+}$is mapped to a point of: (i) the region $D_{\infty}^{+}-$ the trajectory runs to infinity; (ii) the region $D_{n}^{+}$— the trajectory is going to make the next loop; (iii) the line $l_{s}^{+}-$the homoclinic bifurcation occurs. (b) Qualitative behavior of the trajectory forming a heteroclinic linkage and its neighboring trajectories.

the plane $W_{s}\left(P^{ \pm}\right)$and run to infinity, while those going through the region $D_{n}^{ \pm}$remain "below" the plane $W_{s}\left(P^{ \pm}\right)$and after some time will return back to the plane $U^{ \pm}$.

\subsection{Homoclinic and heteroclinic orbits}

\subsubsection{Homoclinic bifurcation}

Consider, first, how the orbit homoclinic to the steady point $P^{+}$is formed in the system (4). It corresponds to the time-dependent solution of the system (4) which asymptotically tends to the steady point as $\xi \rightarrow \pm \infty$. Such solution is the trajectory that simultaneously belongs to the unstable manifold $W_{u}\left(P^{+}\right)$and to the stable manifold $W_{s}\left(P^{+}\right)$of the steady point $P^{+}$. This trajectory should contain the point $M_{u}^{+}$and intersect, at the same time, the line $l_{s}^{+}$. Therefore, the condition of exsistence of homoclinic orbits of the point $P^{+}$can be formulated in the following way. Let the initial conditions of the system (4) be at the point $M_{u}^{+}$. Then, if the flow corresponding to (4) maps the point $M_{u}^{+}$to some point $M_{s}^{+} \in U^{+}$and this point belongs to the line $l_{s}^{+}$, then a homoclinic loop $\Gamma$ appears in the phase space of the system (4).

Let us change the control parameters in a small neighborhood of the point of homoclinicity. Then the mapped point $M_{s}^{+}$can shift from the line $l_{s}^{+}$ either to the region $D_{\infty}^{+}$or to the region $D_{n}^{+}$. In the first case the trajectory started at $M_{u}^{+}$will tend to infinity as shown in Fig. 1 (a) but in the second case this trajectory will go to a small neighborhood of the point $P^{+}$, then intersect the plane $U^{+}$near the point $M_{u}^{+}$, hence the possibility of multi-loop homoclinic orbits near a given loop. According to Shilnikov theorem [Shilnikov, 1969; Shilnikov, 1970] this possibility occurs if the saddle quantity $\sigma$ of the saddle-focus $P^{+}$defined as

$$
\sigma=\lambda_{1}+\max \left\{\lambda_{2}, h\right\}
$$

is positive for the parameters of homoclinicity.

By the earlier mentioned symmetry with the appearance of a loop homoclinic to the steady point 
$P^{+}$, there also appears a loop homoclinic to the steady point $P^{-}$, and the profile of the loop is defined by the transformation (6).

\subsubsection{Heteroclinic bifurcation}

Consider the possibility of heteroclinic orbits formed by the steady points $P^{+}$and $P^{-}$. Such orbits correspond to a solution of the system (4) that simultaneously belongs to the unstable manifold of the point $P^{+}\left[W_{u}\left(P^{+}\right)\right]$and to the stable manifold of the point $P^{-}\left[W_{s}\left(P^{-}\right)\right]$. This condition indicates the solution which contains the point $M_{u}^{+}$and intersects the line $l_{s}^{-}$at some point $M_{s}^{-}$. Thus, the parameters for which the flow (4) maps the point $M_{u}^{+}$to some point $M_{s}^{-} \in U^{-}$which belongs to the line $l_{s}^{-}$correspond to the appearance of a heteroclinic orbit $H_{ \pm}$"linking" the steady points $P^{+}$and $P^{-}$.

Applying the transformation (6) to the orbit $H_{ \pm}$we obtain the heteroclinic orbit $H_{\mp}$ that "started" at the point $P^{-}$and tends to the point $P^{+}$. Thus, there exists a contour $P^{+} \rightarrow H_{ \pm} \rightarrow$ $P^{-} \rightarrow H_{\mp} \rightarrow P^{+}$in the phase space of the system (4) [see Fig. 1(b)] [Turaev, 1996; Shashkov \& Turaev, 1996]. We show below that this contour is associated with the existence in the medium of solitary pulses originated from two fronts (kinks) each of which is described by the heteroclinic orbits $H_{ \pm}$ and $H_{\mp}$ taken separately.

\subsection{Bifurcation set}

To define the parameters corresponding to the homoclinic and heteroclinic bifurcation for fixed $k=k^{*}$, we calculate the split function which is

$$
S_{\Gamma}(\alpha)=\operatorname{dev}\left(M_{s}^{+}, l_{s}^{+}\right)
$$

for a homoclinic loop $\Gamma$, and

$$
S_{H_{ \pm}}(\alpha)=\operatorname{dev}\left(M_{s}^{-}, l_{s}^{-}\right)
$$

for a heteroclinic orbit $H_{ \pm}$. The sign dev denotes the deviation of a point from a line. The parameter $\alpha^{*}$ corresponding to a zero of the split function determines the bifurcation point $\left(\alpha^{*}, k^{*}\right)$ in the state space $(\alpha, k)$. The location of the lines $l_{s}^{ \pm}$and the points $M_{u}^{ \pm}$is obtained analytically as it has been done in [Nekorkin et al., 1995]. The map $M_{u}^{+} \rightarrow M_{s}^{ \pm}$is determined by numerical integration of the system (4).

Let us turn to Fig. 2 where the bifurcation diagram is displayed. We denote the bifurcation curves by the letters $\Gamma$ and $H$. A superscript $n$ indicates the number of loops made by the orbit and the subscript $s$ or $l$ characterizes the "magnitude" of the pulse (" $s$ " - small, "l" - large). Figure 2(a) shows the diagram in rather large scale in $(\alpha, k)$ plane. Two close curves $\Gamma_{l}^{1}$ and $H_{l}^{1}$ correspond to the simplest types of homoclinic and heteroclinic orbits. The shapes of the orbits in the phase space and their profiles $(x(\xi))$ are displayed in Fig. 3 and are marked by the same letters as the corresponding bifurcation curves (see Fig. 3(a) for profiles of the simplest orbits $\Gamma_{l}^{1}, H_{l}^{1}$ and $\Gamma_{s}^{1}$ ). For large enough values of $k$ there exists a number of homo- and heteroclinic bifurcations corresponding to the pulses of "small magnitude" (the curves marked by letters $H_{s}^{n}$ and $\Gamma_{s}^{n}$ in Fig. 2). ${ }^{2}$ As earlier said " $n$ " indicates the number of turns made by the orbits near the steady point $O$. Some profiles of multi-rotated orbits are given in Fig. 3(b).

The dashed curve in Fig. 2 corresponds to the bifurcation of the equilibrium $O$, hence this point becomes a stable focus (to the right of the dashed curve) with two pairs of complex eigenvalues. In this region the trajectory $W_{u}\left(P^{+}\right)$(see Fig. 1) is attracted by the stable point $O$ hence multi-rotated homoclinic orbits do not appear for such parameters. In this case the solution formed by $W_{u}\left(P^{+}\right)$ is also bounded ("connects" the points $P^{+}$and $O$ ) hence corresponds to a possible travelling wave in the array (1). However, as earlier noted the homogeneous equilibrium state of the array corresponding to the point $O$ is unstable. Then such wave solutions are also unstable.

Having calculated the saddle quantity $\sigma$ of the saddle-focus $P^{+}$we find that it has a positive value for each point of all bifurcation curves associated with single-loop homoclinic bifurcation $\left(\Gamma_{l}^{1}, \Gamma_{s}^{1}, \Gamma_{s}^{2}, \Gamma_{s}^{3}, \ldots\right)$. Hence, according to the Shilnikov theorem for the parameters varying in a small neighborhood of each curve there occurs a number of homoclinic bifurcations resulting in the appearance of multi-loop homoclinic orbits. Besides, the neighborhood of the homoclinicity in the phase space contains a countable set of hyperbolic

\footnotetext{
${ }^{2}$ We distinguish the large and small orbits in the following way. Each loop of the large orbit of $P^{+}$extends to the "opposite" outer linear region intersecting both planes $U^{-}$and $U^{+}$, while the small loops have intersections with the plane $U^{+}$only.
} 


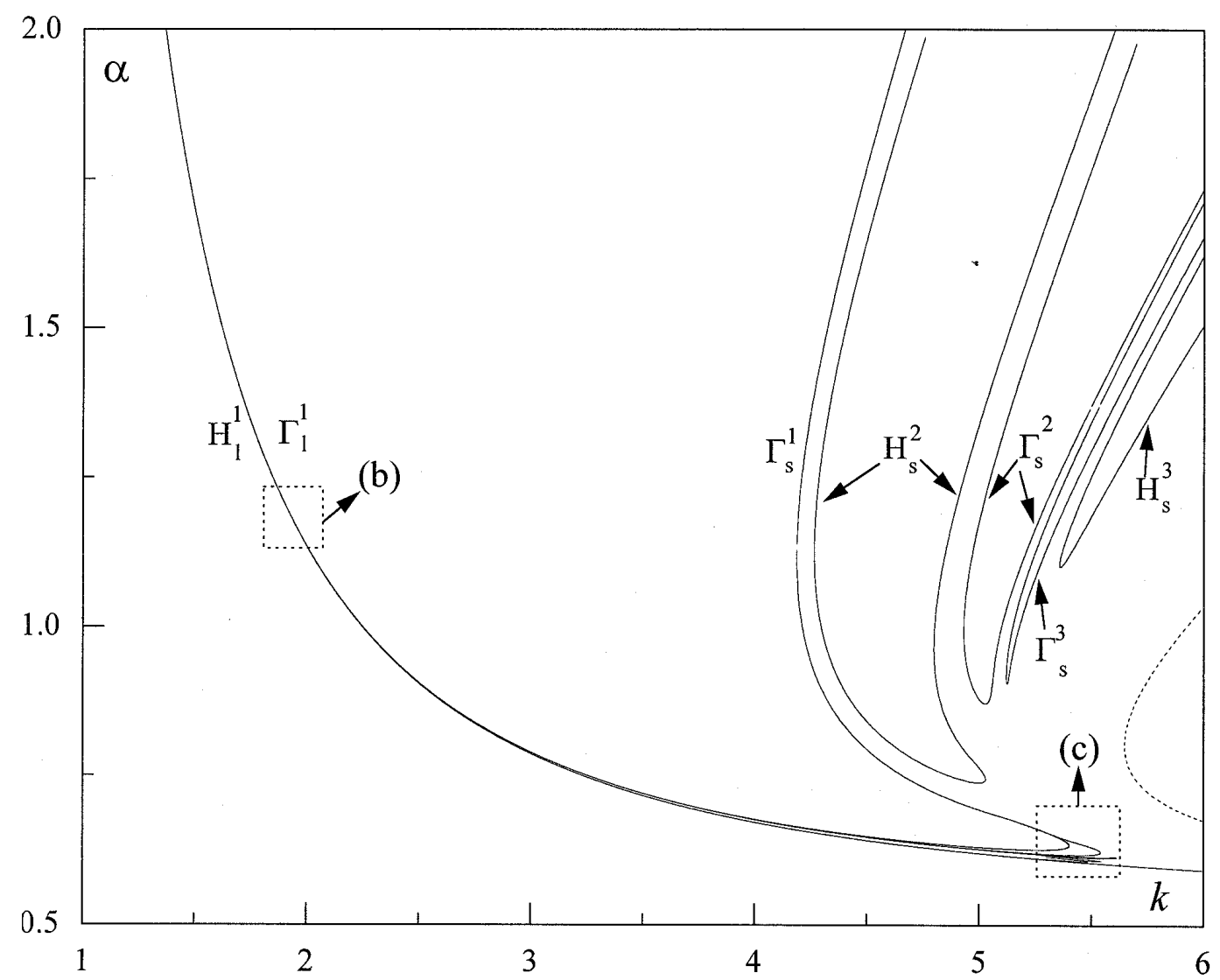

(a)

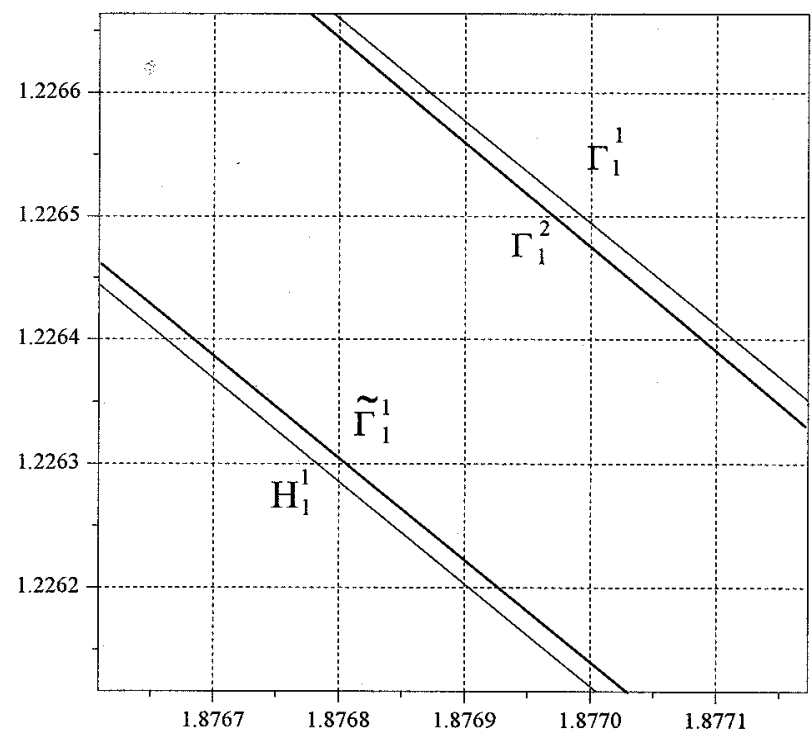

(b)

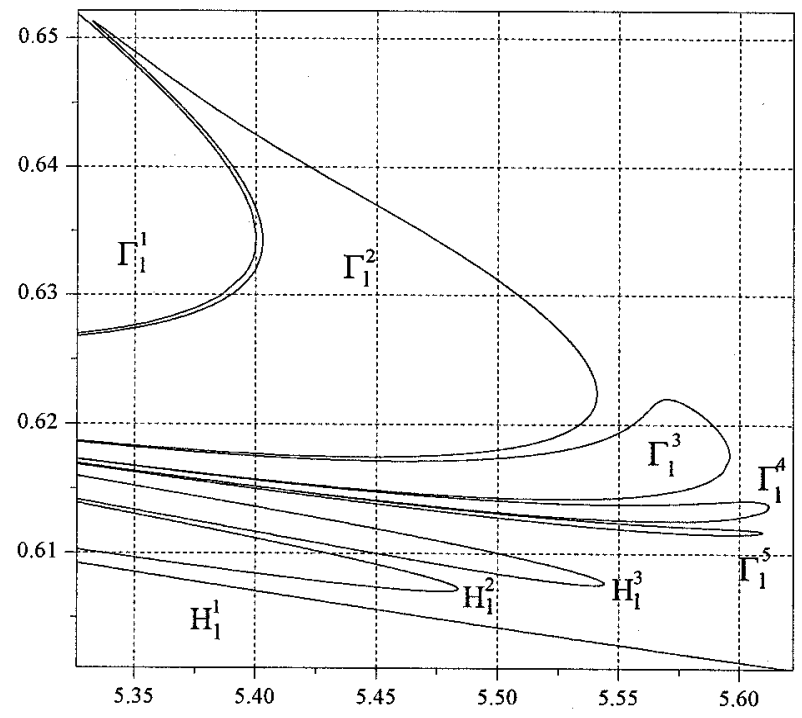

(c)

Fig. 2. Bifurcation diagram for homoclinic $\Gamma_{l, s}^{n}$ and heteroclinic $H_{s, l}^{n}$ orbits in the $(k, \alpha)$ parameter plane. The superscript $n$ indicates the number of rotations or loops made by an orbit in the phase space (or the number of humps along the profile of the orbit). The subscript $s$ or $l$ distinguishes the orbits with all loops of large "magnitude" and those containing at least one loop of small "magnitude". (a) Full diagram. (b) and (c) Two enlarged regions. 

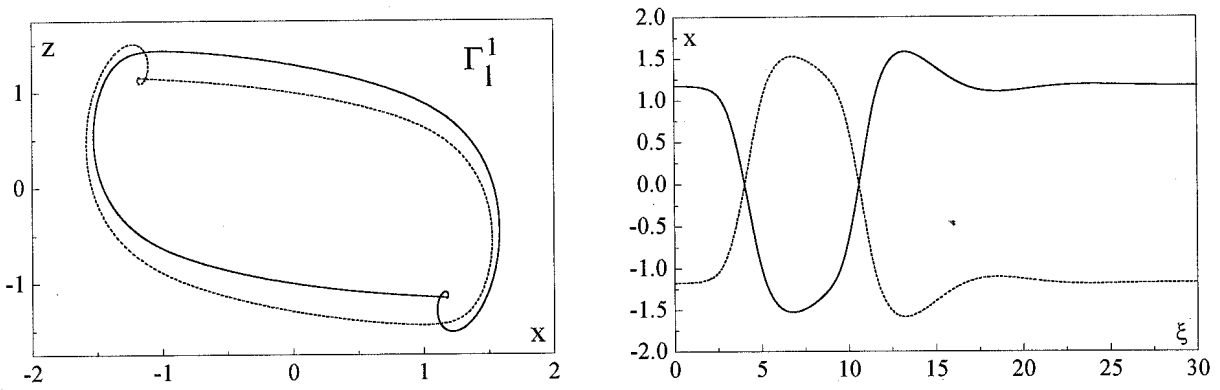

(a1)
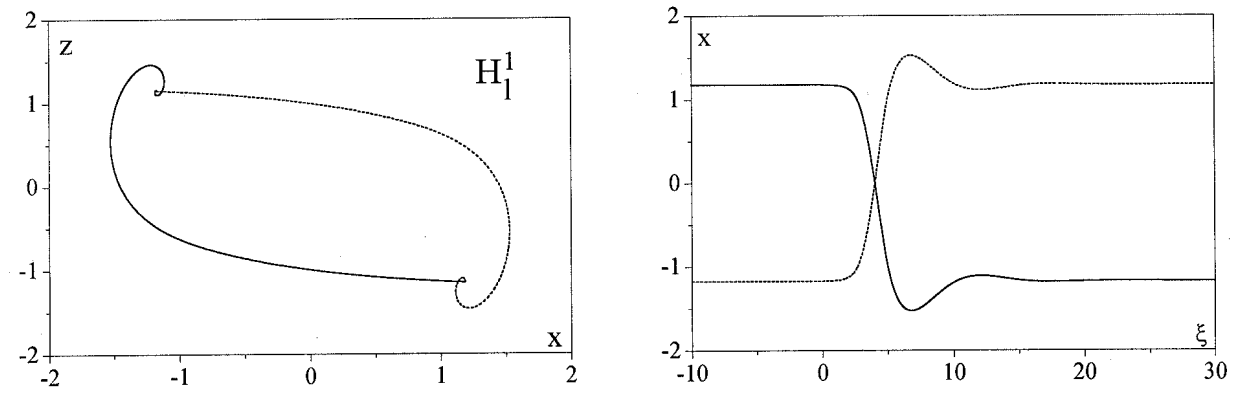

(a2)
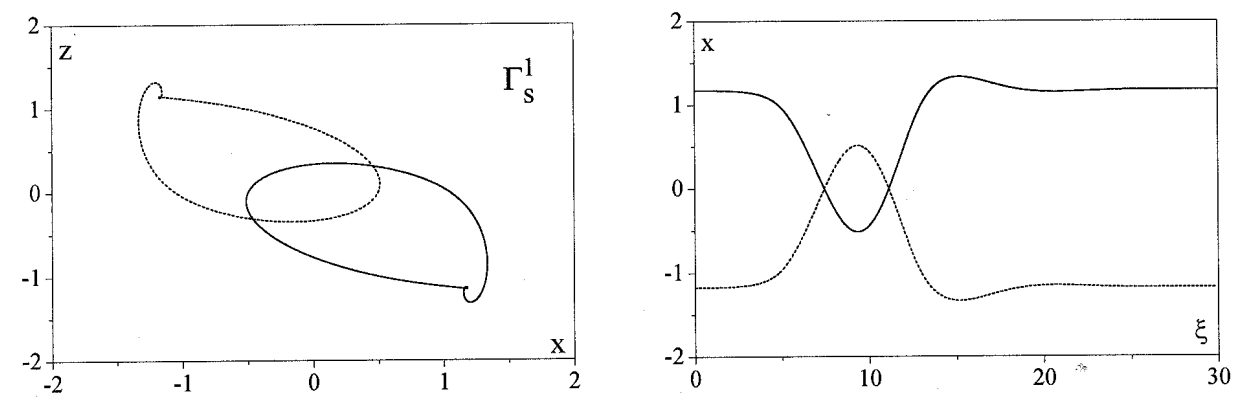

(a3)
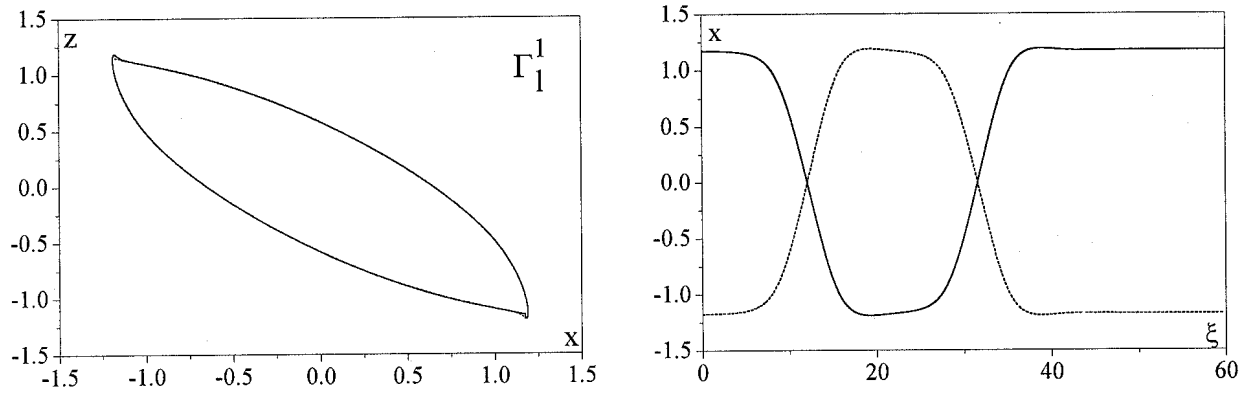

(a4)

Fig. 3. Orbits in phase space and their corresponding profiles for different points of the bifurcation diagram. (a) The simplest, single-loop homoclinic and heteroclinic orbits. (a1) The orbit $\Gamma_{l}^{1}$ for $\alpha=1.500, k=1.624$; (a2) The orbit $H_{l}^{1}$, $\alpha=1.502, k=1.622 ;$ (a3) The orbit $\Gamma_{s}^{1} ; \alpha=1.501, k=4.324 ;$ (a4) The orbit $\tilde{\Gamma}_{l}^{1}, \alpha=0.610, k=5.300$. Dashed curves show the symmetric orbits that also exist in the system (4). (b) Multi-rotated orbits of small "magnitude" and corresponding wave profiles. (b1) $\Gamma_{s}^{2}, \alpha=1.503, k=5.576$; (b2) $H_{s}^{2}, \alpha=1.502, k=4.405$; (b3) $\Gamma_{s}^{3}, \alpha=1.502, k=5.706$; (b4) $H_{s}^{3}$, $\alpha=1.500, k=5.824$. (c) The profiles of multi-looped orbits of large "magnitude". (c1) $\Gamma_{l}^{2}, \alpha=0.620, k=5.304 ;(\mathrm{c} 2) \Gamma_{l}^{3}$, $\alpha=0.618, k=5.301 ;(\mathrm{c} 3) \Gamma_{l}^{4}, \alpha=0.618, k=5.302 ;(\mathrm{c} 4) \Gamma_{l}^{5}, \alpha=5.301, k=0.618 ;(\mathrm{c} 5) H_{l}^{2}, \alpha=0.615, k=5.300 ;(\mathrm{c} 6) H_{l}^{3}$, $\alpha=0.617, k=5.301$. 

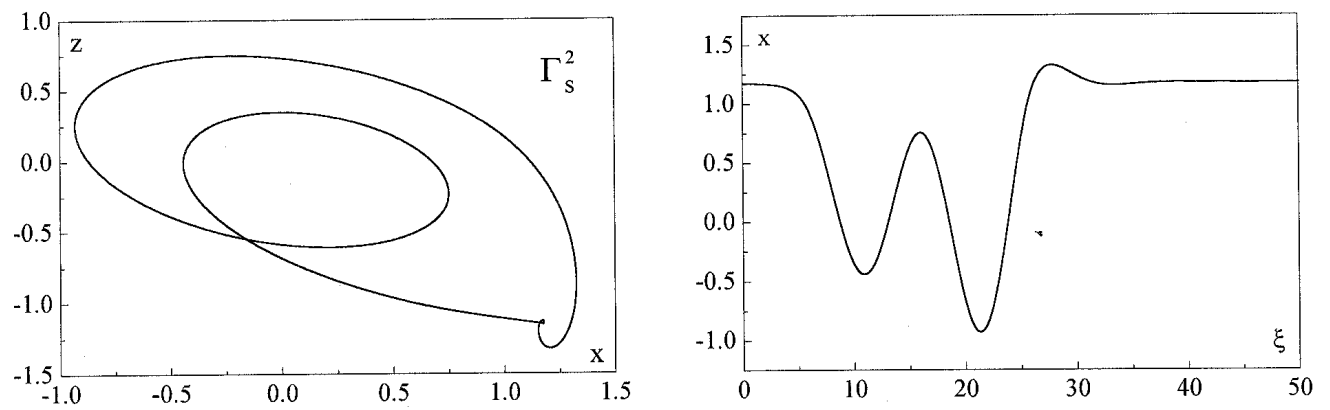

(b1)
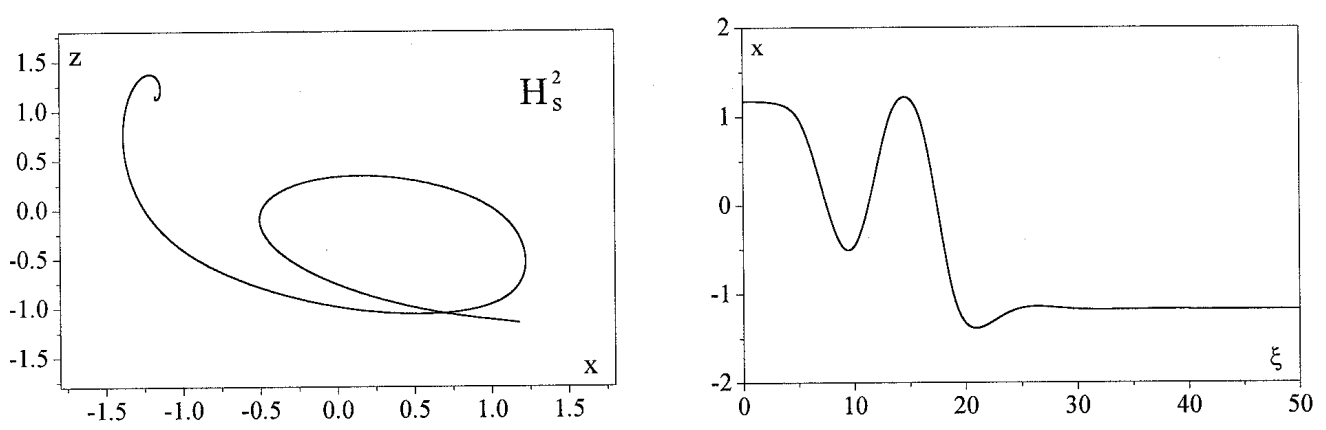

(b2)
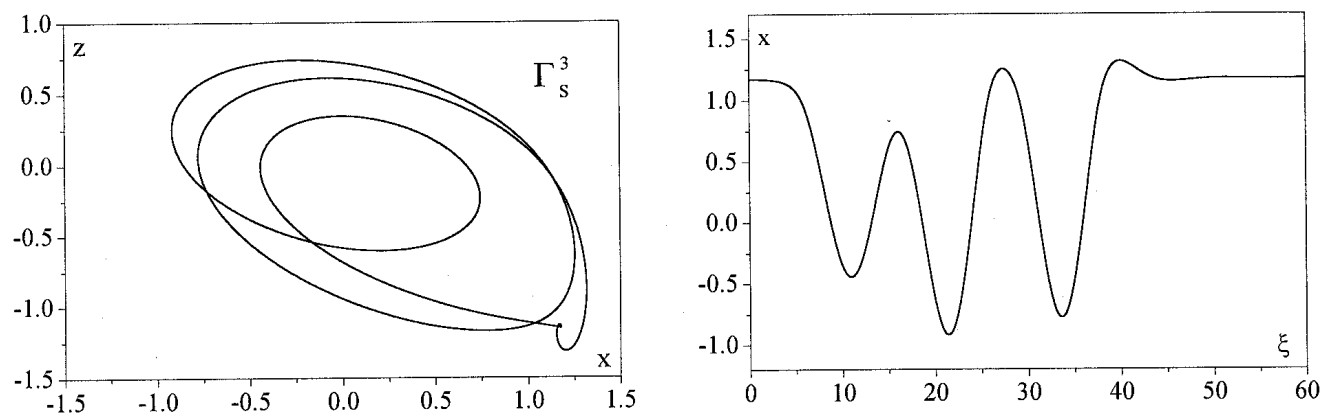

(b3)
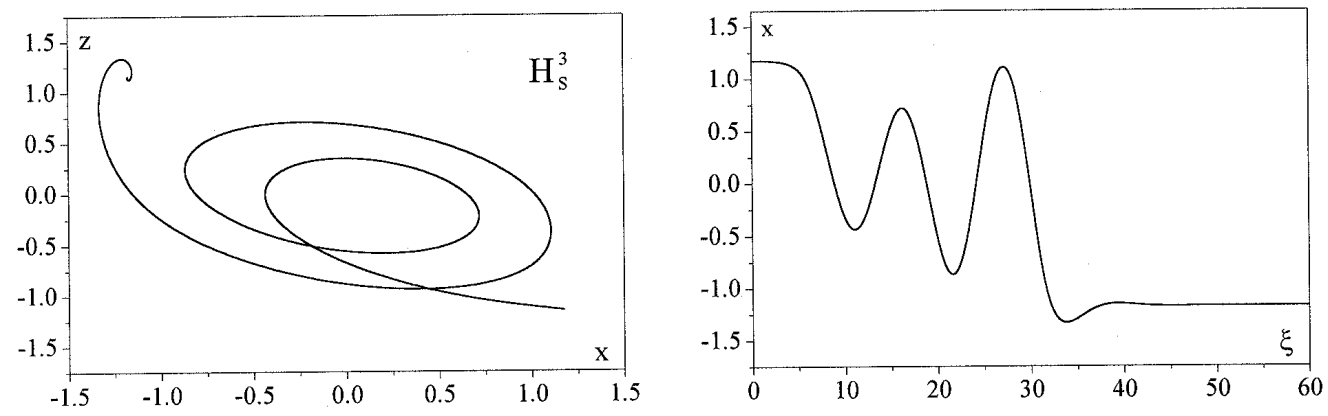

(b4)

Fig. 3. (Continued) 


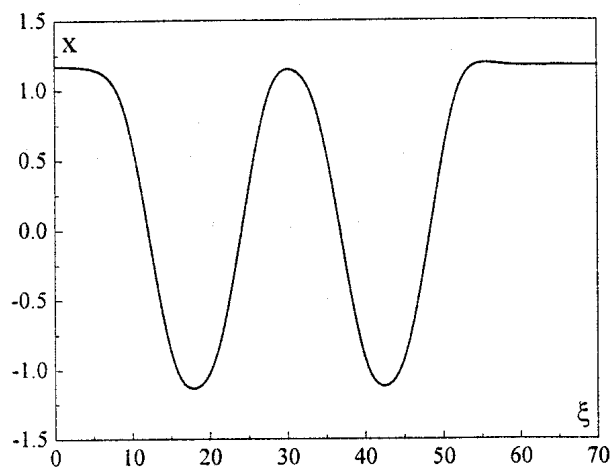

(c1)

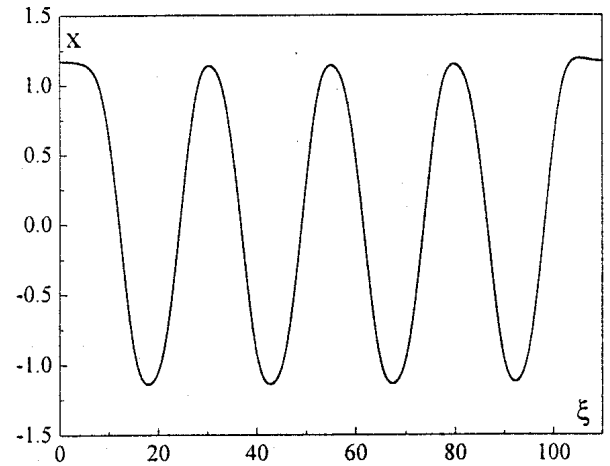

(c3)

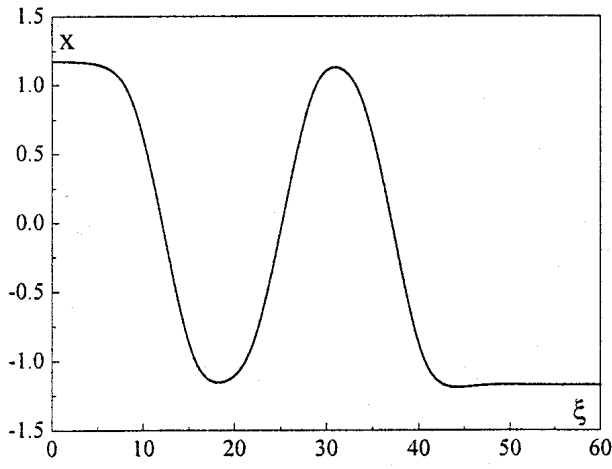

(c5)

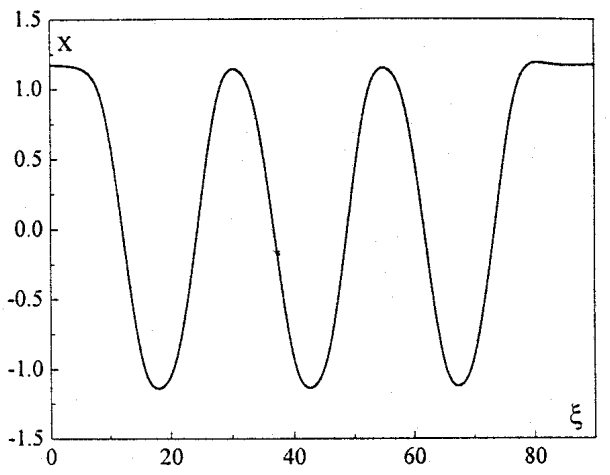

(c2)

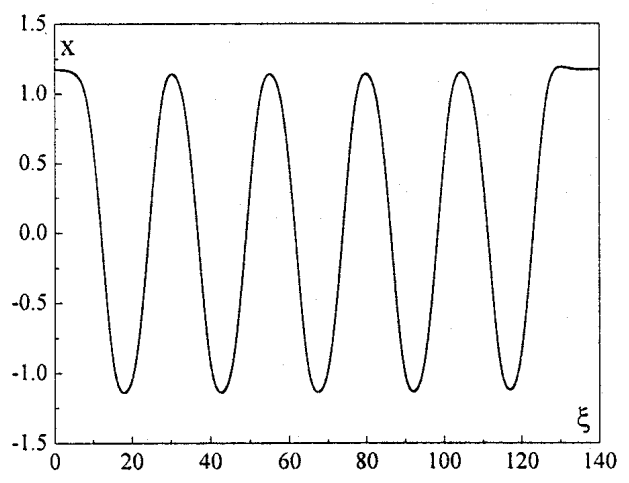

(c4)

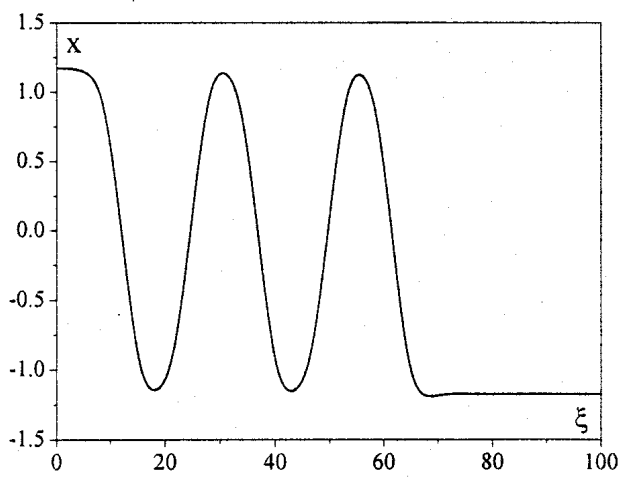

(c6)

Fig. 3. (Continued)

periodic orbits. Figures 2(b) and 2(c) present two enlarged regions taken near the bifurcation curve $\Gamma_{l}^{1}$. Here different multi-loop orbits can be distinguished. The profiles of the multi-loop orbits are depicted in Fig. 3(c).

As it has been earlier mentioned the heteroclinic bifurcation $H_{l}^{1}$ yields the appearance of the heteroclinic contour in the phase space of the system (4). We find that the neighborhood of the curve $H_{l}^{1}$ contains a number of bifurcation points which correspond to the appearance of single- and multiloop homoclinic orbits $\tilde{\Gamma^{n}}$. The profile of each loop of these orbits is "constructed" from two symmetric heteroclinic orbits taken from the contour [see the simplest one $\tilde{\Gamma}^{1}$ in Fig. 3(a)]. Besides, there exist solutions having the form of multi-loop heteroclinic orbits. Figure 3(c) illustrates the examples of such orbits for $n=2,3$ loops.

In summary: The fourth order system (4) describing the profiles of possible travelling waves 
in the array (1) allows a wide variety of bounded solutions. These are

- Single- and multi-loop homoclinic orbits with different "magnitude";

- Multi-rotated homoclinic orbits;

- Heteroclinic orbits of different form;

- Homoclinic solutions arising from two symmetric heteroclinic orbits;

- Periodic orbits of hyperbolic type with quite complex profile (that occur near each homoclinicity).

Each of these solutions ensures the existence of a wave with a definite profile which propagates along the array with constant velocity $c=1 / h$. However, the investigation of the system (4) does not ensure their stability.

\section{Excitation and Evolution of Solitary Pulses and Fronts}

Let us assume that the discrete medium modeled by (1) initially has been in a stable equilibrium associated with the homogeneous state $P^{+}$. How should we perturb this state to observe, for example, a travelling pulse associated with an orbit of the system (4) homoclinic to the steady point $P^{+}$? As earlier shown, near a given loop there exist multi-loop orbits whose profiles determine the multi-hump pulses. Thus, for a given set of parameter values there can propagate a number of different travelling waves. To differentiate these waves we construct the initial excitation of the state $P^{+}$ as close as possible to the profile of a given homoclinic orbit. If the pulse is stable we will observe in the simulation the steady translation of the initial profile along the array. If this pulse is unstable it will go to another attractor of the system (1).

\subsection{Construction of the wave profiles}

Let us take a point $\left(\alpha^{*}, k^{*}\right)$ of the bifurcation curve corresponding, for instance, to a homoclinic orbit $\Gamma_{l}^{1}$. The space profile of the solitary pulse associated with this orbit can be constructed by using (3) with $\xi=j h$ from the profile of the homoclinic orbit $(x(\xi), y(\xi), z(\xi))$. Let us take the core of this profile of "length" $T\left(\xi \in\left[\xi_{0}, \xi_{0}+T\right]\right)$ that satisfies the condition

$$
\begin{gathered}
\left|x(\xi)-x_{0}\right| \ll 1, \quad\left|y(\xi)-y_{0}\right| \ll 1, \\
\left|z(\xi)-z_{0}\right| \ll 1 \\
\forall \xi<\xi_{0}, \quad \xi>T+\xi_{0} .
\end{gathered}
$$

Hence the "tails" of the pulse are close enough to the steady state $P^{+}$.

Then, we perturb the homogeneous stable state $P^{+}$by "forcing" $N_{1}$ elements $\left(N_{1}=T / h\right)$ in the array starting from the element $k_{0}$ and mimicking the profile of the homoclinic orbit

$$
\begin{aligned}
& x_{k+k_{0}}(t=0)=x\left(\xi_{0}+k h\right), \\
& y_{k+k_{0}}(t=0)=y\left(\xi_{0}+k h\right), \\
& z_{k+k_{0}}(t=0)=z\left(\xi_{0}+k h\right)
\end{aligned}
$$

with $k=1, \ldots, N_{1}$. We use such space distribution as the initial condition for the numerical simulation of the system (1) to study the evolution of travelling waves.

Note, that to satisfy the continuum approximation which we used to obtain the system (4), the parameter $h$ should be chosen small enough $(h \ll T)$ to ensure the necessary smoothness of the wave profile. Then, the value of the diffusion coefficient $d$, $d=\frac{k^{*}}{h^{2}}$, appears rather large.

\subsection{Stable and unstable solitary pulses}

Figures 4 illustrate the evolution of the solitary pulses associated with the homoclinic orbits of large "magnitute" (obtained from the curves $\Gamma_{l}^{n}$ of the bifurcation diagram). Figure 4(a) shows a single pulse (orbit $\Gamma_{l}^{1}$ ), Fig. 4(b) - a two-humps pulse (orbit $\Gamma_{l}^{2}$ ) and Fig. 4(c) - a five-humps pulse (orbit $\Gamma_{l}^{5}$ ). With the initial distributions close to the homoclinic orbit profile in the way described above we observe the solutions which travel along the array with constant velocity and no apparent change of form. For zeroflux boundary conditions the pulses are absorbed by the boundary and the terminal state (as $t \rightarrow \infty$ ) of the array becomes the homogeneous state $P^{+}$. The circular array exhibits stable pulses propagating around the circle. These pulses represent waves of cnoidal type whose images in the phase space of the system (4) are hyperbolic periodic orbits which, according to Shilnikov theorem occur near homoclinicity with positive saddle quantity.

Note, that due to symmetry there can propagate solitary pulses of inverse profiles relative to the "background" homogeneous state $P^{-}$. In the terminology used in the field of optical fibers [Hasegawa \& Kodama, 1995; Huang \& Velarde, 1996] we can say that the discrete medium (1) allows the propagation of both "bright" and "dark" pulses of wide variety of spatial forms. 


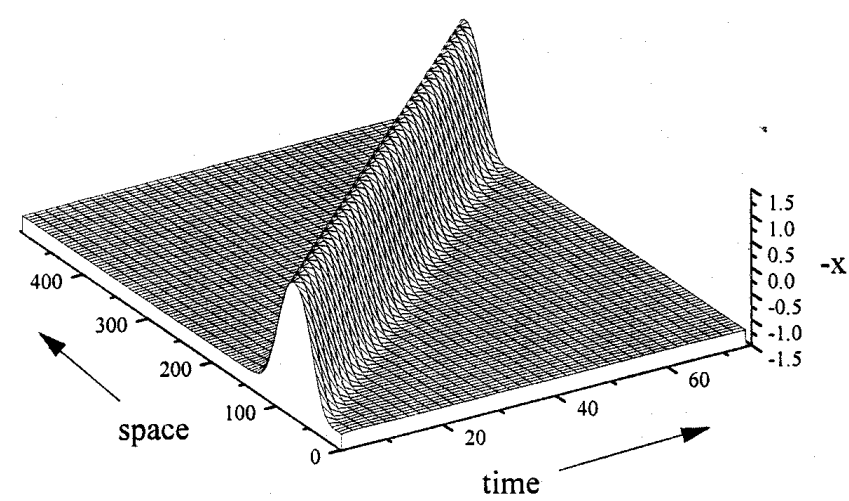

(a)

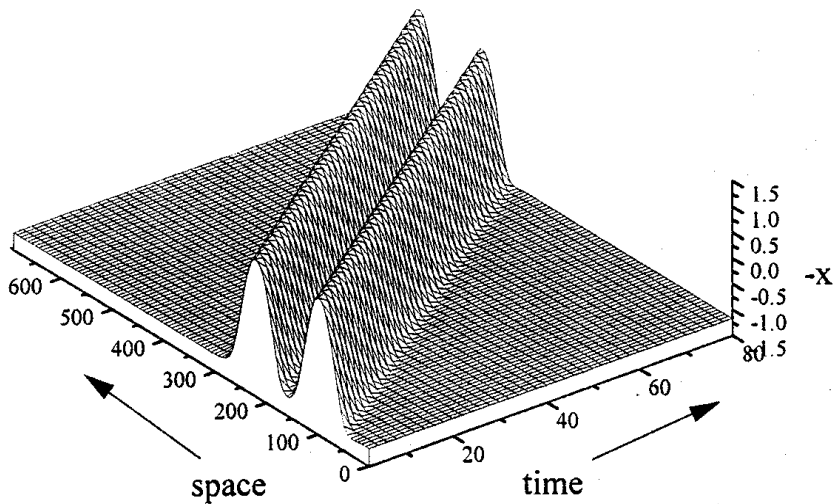

(b)

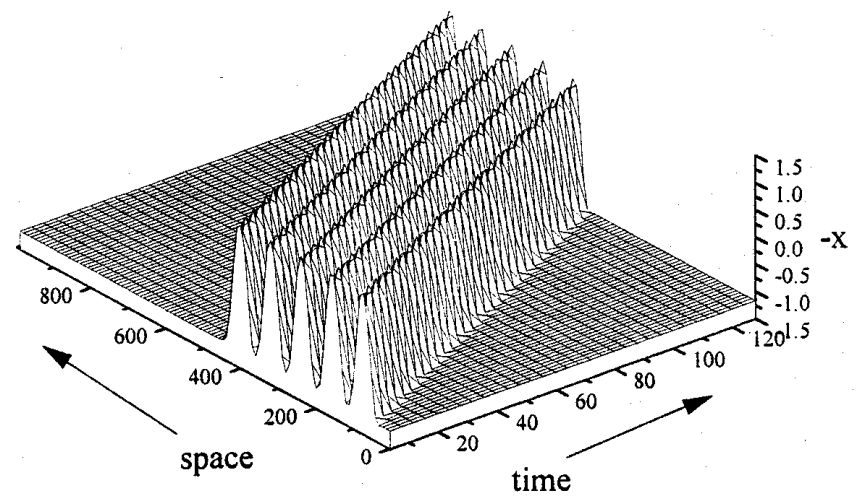

(c)

Fig. 4. Space-time evolution of stable, large "magnitude" pulses. For the numerical simulation of the discrete medium, the array has been initially forced by a disturbance close to the profile of the homoclinic orbits of the system (4). (a) Single-hump pulse, $\alpha=0.627, d=133$. (b) Two-hump pulse, $\alpha=0.620, d=133$. (c) Five-hump pulse, $\alpha=5.301, d=59$.

Consider, now, the behavior of solitary pulses associated with homoclinic orbits of a small "magnitude" (curves $\Gamma_{s}^{n}$ in Fig. 2). All pulses of such type appear unstable in the numerical simulation of the array (1). Figure 5 illustrates, the two ways of evolution of the single pulse of the small amplitude $\Gamma_{s}^{1}$ for two parameter sets. The first case provides the decay of the small pulse to the "background" homogeneus state $P^{+}$[Fig. 5(a)], but in the second one the pulse evolves to the stable wave of large "magnitude" [Fig. 5(b)] which is associated with the homoclinic orbit $\Gamma_{l}^{1}$. It means that depending on parameter values the initial excitation can fall either into the basin of attraction of the homogeneous, stable steady state $P^{+}$, or in the stable solitary pulse.

Note, that the solitary pulses of large "magnitude" could be excited from many initial disturbances. Figure 5(c) illustrates the appearance of two solitary pulses from rather simple initial conditions (a part of the elements has been initially at the state $P^{+}$, and the other part at the state $P^{-}$).

\subsection{Wave fronts and pulses constructed from two fronts}

Together with the single and multi-hump pulses, the array (1) can exhibit other travelling wave fronts (kinks) associated with the heteroclinic orbits of the system (4). These solutions can be interpreted as the transient process in the "selection" of a terminal stable homogeneous state, $P^{+}$or $P^{-}$. The evolution of the single and two-hump fronts is illustrated in Figs. 6(a), 6(b). The profiles of these fronts are derived from the heteroclinic orbits $H_{l}^{1}, H_{l}^{2}$. The fronts whose profiles contain humps of small "magnitude" (curves $H_{s}^{n}$ of Fig. 2) become unstable and evolve to the simplest front $\left(H_{l}^{1}\right)$, i.e. they 


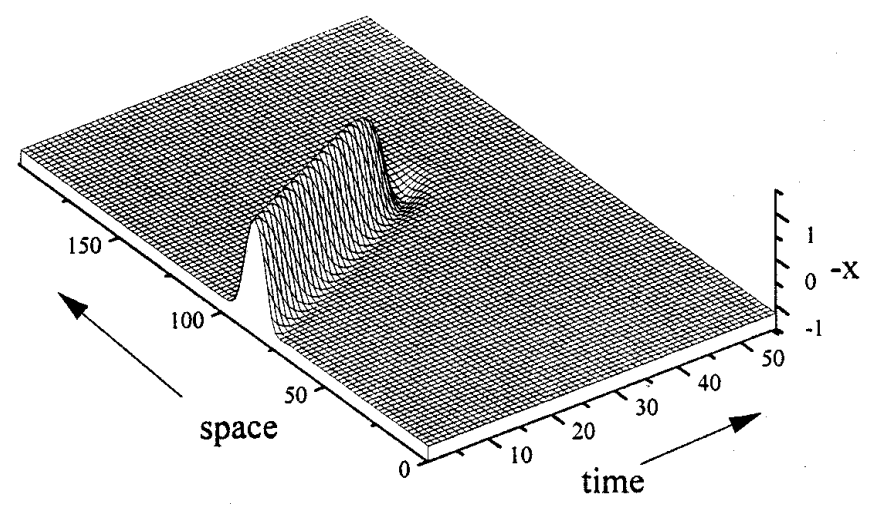

(a)

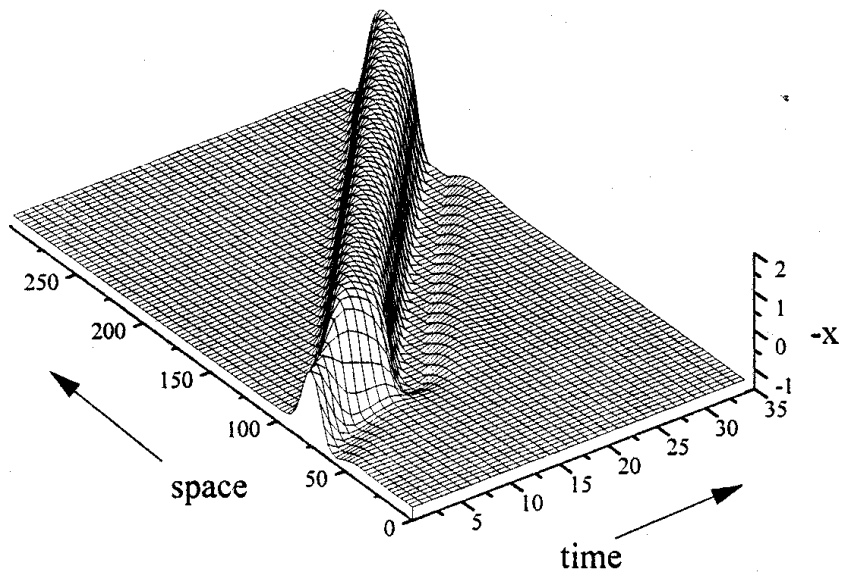

(b)

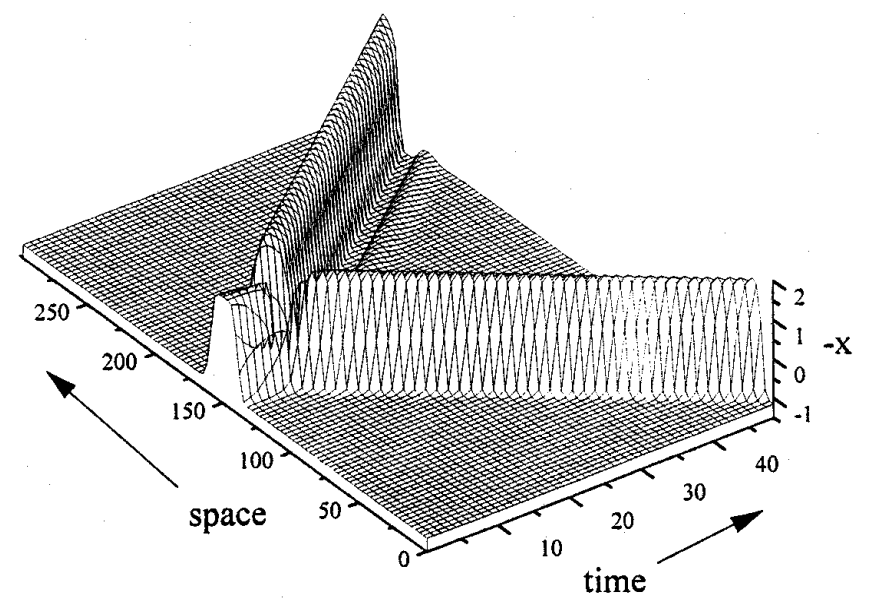

(c)

Fig. 5. Evolution of the initial condition derived from the homoclinic orbits of small "magnitude". (a) Decay of the pulse to the "background" steady state. Parameter values: $\alpha=0.66, d=6.5$. (b) Transformation of a small "magnitude" pulse to a stable pulse large "magnitude". Parameter values: $\alpha=2.00, d=52$. (c) Onset of two stable pulses traveling in opposite directions from initial conditions taken in the form of pedestal. Parameter values: $\alpha=3.74, d=10$.

are unstable like the solitary pulses of small "magnitude" [see, e.g. Fig. 6(c)].

As predicted in the previous section, pulses whose profiles are composed of two symmetric fronts can be propagated. The initial conditions for such waves can be derived from a homoclinic orbit $\tilde{\Gamma}_{l}^{1}$ or in a simple way by taking the two symmetric kinks with a finite delay. Such fronts are stable if the constituent fronts are stable. Figure 6(d) illustrates the evolution of a pulse composed of two stable two-hump fronts.
In summary, the discrete medium modeled by the system (1) allows stable wave solutions of extremely diverse profiles including "dark" and "bright" pulses of single and multi-hump forms, kinks and "anti"-kinks (associated with symmetric heteroclinic orbits), multi-hump fronts and pulses composed of several set of fronts. Note, that all this diversity of wave motions could be realized for the same parameter values of the array (1), and all waves would propagate with almost equal velocities. $^{3}$

\footnotetext{
${ }^{3}$ For a given $d>d_{0}$ there exists $h_{i}<h_{0}$ to hit any bifurcation point $\left(\alpha^{*}, k_{i}^{*}\right)$ corresponding to $i$ th type of travelling waves. For the large "magnitude" waves all $k_{i}$ will be localized between the curves $H_{l}^{1}$ and $\Gamma_{l}^{1}$ for the fixed $\alpha=\alpha^{*}$ (see bifurcation diagram Fig. 2).
} 


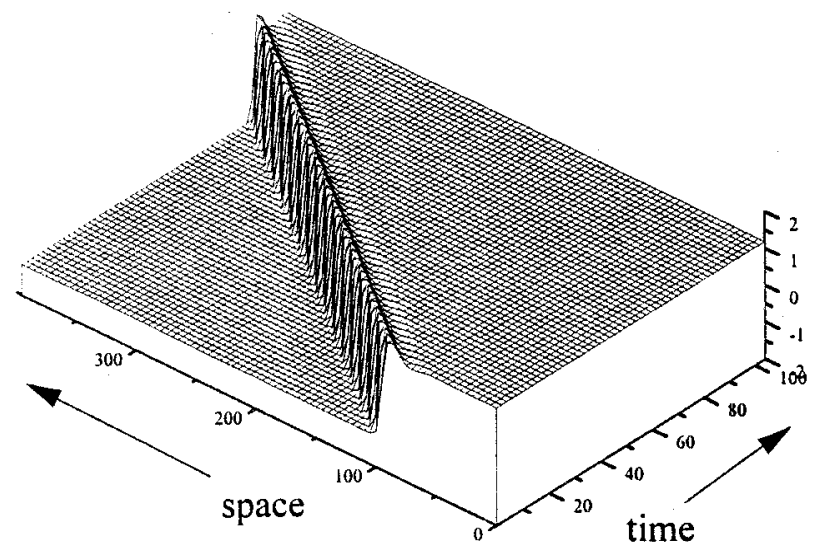

(a)

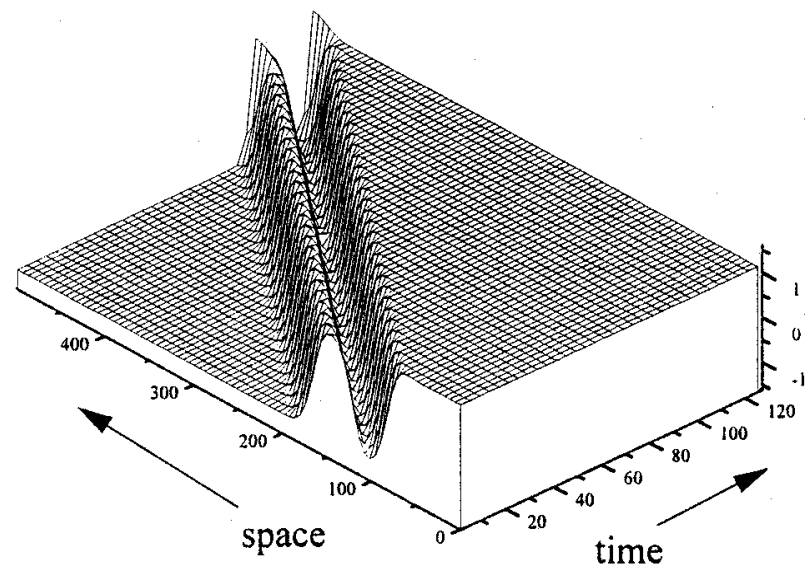

(c)

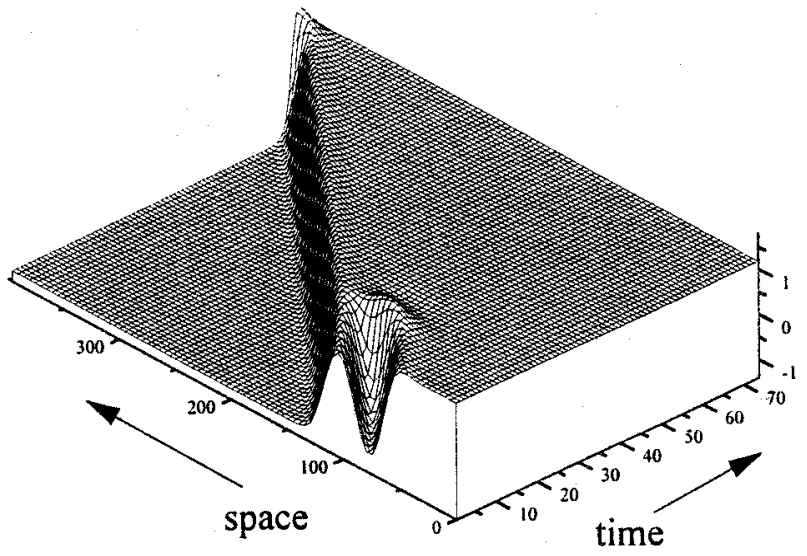

(b)

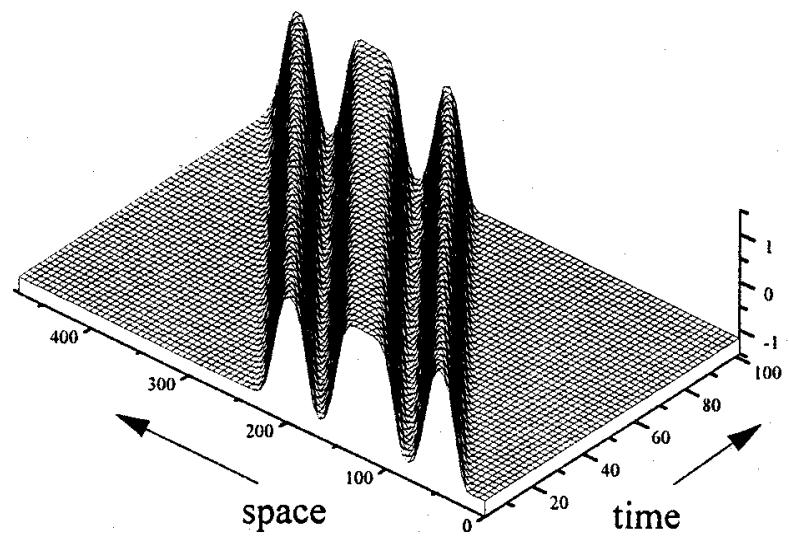

(d)

Fig. 6. Space-time evolution of wave fronts whose profiles have been derived from the heteroclinic orbits of the system (4). The fronts provide the "selection" of the terminal $(t \rightarrow \infty)$ homogeneous steady state of the array $P^{+}$or $P^{-}$. (a) Simple wave front associated with the orbit $H_{l}^{1}$. Parameter values: $\alpha=2.00, d=15.2$. (b) Stable multi-hump wave front (orbit $H_{l}^{2}$ ). Parameter values: $\alpha=0.615, d=59$. (c) Transformation of the unstable wave front associated with the orbit $H_{s}^{2}$ to the simplest front (orbit $H_{l}^{1}$ ). Parameter values: $\alpha=0.755, d=56$. (d) Stable localized structure consisting of two stable multi-hump fronts. It can be interpreted as the multi-hump pulse associated with a homoclinic orbit from the family $\tilde{\Gamma}_{l}^{n}$. Parameter values: $\alpha=0.615, d=33$.

\section{Construction of Chaotic Wave Trains}

Let us now show how the discrete medium (1) allows the propagation of wave trains of extremely complex profile even with disordered or chaotic configuration.

Let us note, first, that the "tails" of all stable pulses and fronts decay rather quickly along the spatial coordinates. As it has been earlier mentioned the velocities of all large "magnitude" waves are close to each other. Then, if in the array we put two different pulses (for example, a single pulse and a two-hump pulse) with a finite, rather long delay, they can form a bound state as a result of a weak interaction between the initial pulses. This state represents the three-hump pulses traveling with constant velocity (not equal, but close to the velocity of the initial pulses) (see e.g. [Christov \& Velarde, 1995]). In the phase space of the system (4) this state appears as a three-loop homoclinic 
orbit (or "three-loop" periodic orbit for the circular array). Its existence near the loops with a positive saddle quantity is ensured by the Shilnikov theorem. Complex wave trains composed of one- and two-hump pulses are shown in Fig. 7(a). Numerical integration of the array (1) shows the stability of this train as expected. Indeed the wave "consists of" two stable pulses.
Similarly, more complex wave trains can be constructed. Consider a rather long array composed of numerous elements. Building the wave train from a large number of stable pulses and fronts, which can propagate separately, we obtain a wave of rather complex profile. Note, that the delay or the distance in space between each neighboring components could vary in very wide limits.

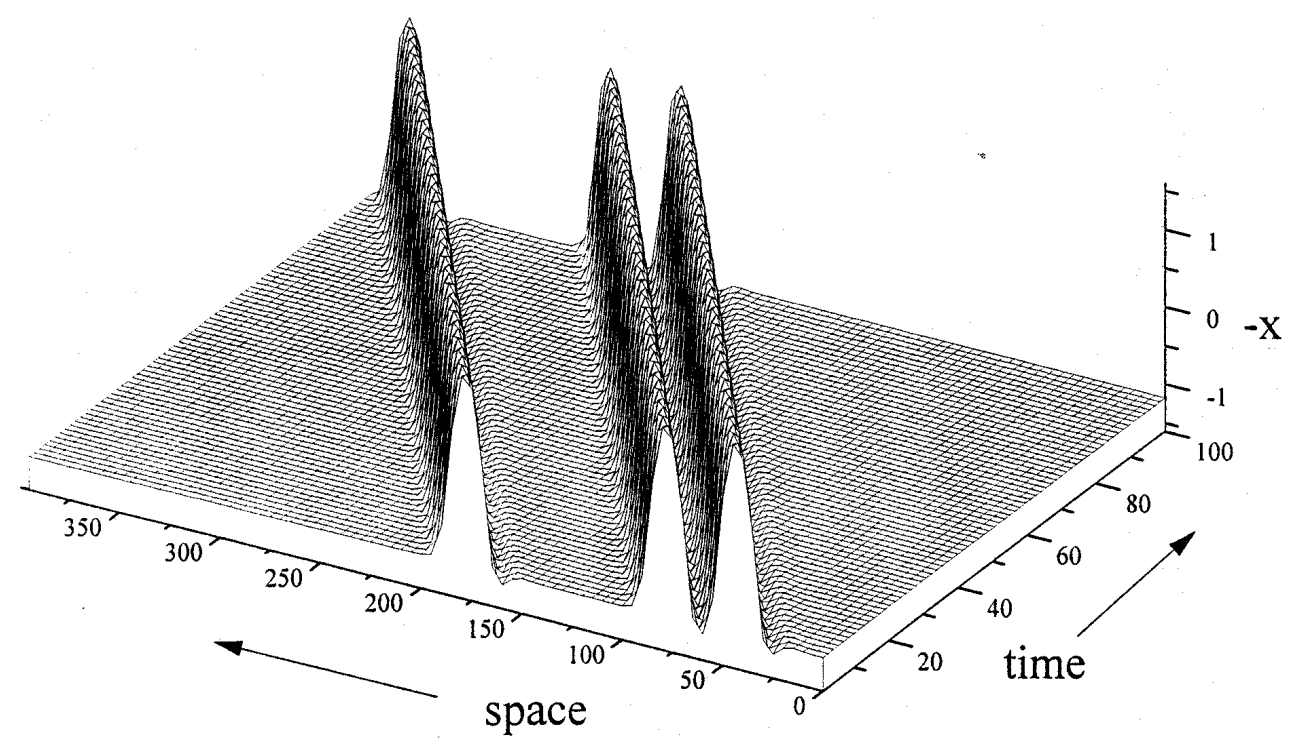

(a)

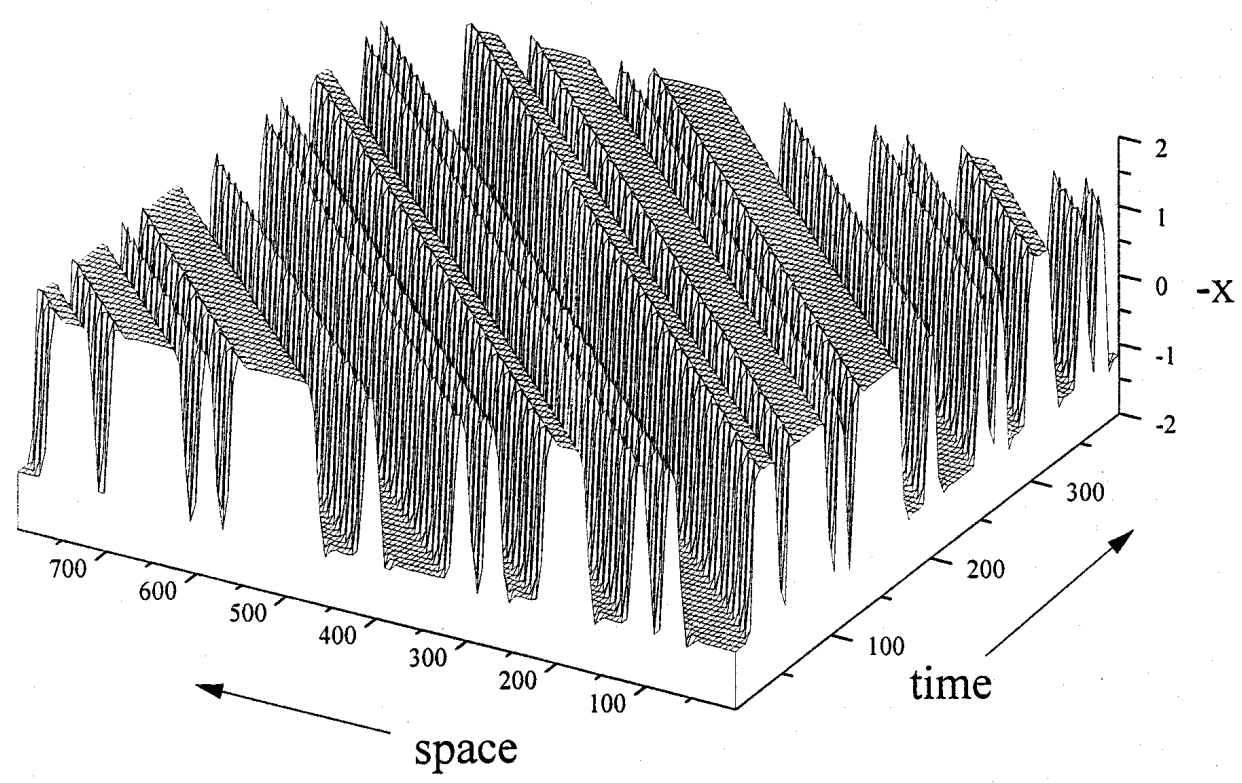

(b)

Fig. 7. Wave trains composed of a sequence of stable pulses and fronts for the parameters taken near $\alpha=0.8, d=11.75$. (a) Interaction of single- and two-hump pulses ends up in a stable bound state. (b) Complex wave train composed of a disordered sequence of stable pulses and fronts. Its evolution corresponds to the numerical simulation in the circular array. 
Extending the boundaries of the array to infinity we can speak about the excitation of chaotic wave trains in the system. These are profiles composed of a disordered sequence of pulses and fronts. Such a sequence can be associated with the homoclinic (heteroclinic) orbit of an infinite number of loops of the system (4).

To investigate the evolution of chaotic trains we use a circular array with a large enough number of elements. In this case the disordered train appears in the phase space (4) as a complex periodic orbit from the countable set (see Sec. 4). Putting in the array, the initial condition in the form of an arbitrary sequence of pulses and fronts, we find that it tends to the stable solution steadily translating along the array [see Fig. 7(b)], i.e. hence a stationary wave of complex profile.

In summary, the discrete medium modeled by (1) can sustain chaotic wave trains.

\section{Conclusion}

We have shown that a one-dimensional lattice of Chua's circuits allows a wide variety of stable traveling wave solutions, from a simple solitary pulse or a wave front to quite complex long wave trains, "bright" and "dark" (optical) pulses or chaotic sequences of pulses and fronts. The lattice represents, in fact, a discrete medium whose properties are quite similar to a continuum, reaction-diffusion system with one diffusing component.

We have studied the possible traveling waves and their stability by analyzing the underlying fourth-order system of ODEs whose bounded solutions correspond to the profiles of the waves. Illustration of the actual wave profiles and wave trains found has been done by numerically simulating the dynamical system.

In conclusion, our results show the possibility of using a lattice of Chua's circuits as a tool for the development of information transmission channels or for modeling wave processes in different areas of science and technology.

\section{Acknowledgments}

Part of this research was carried out while V. I. Nekorkin held a Sabbatical position with the Instituto Pluridisciplinar at the Universidad Complutense. This research was supported by NATO under Grant OUTR. LG 96-578, by DGICYT
(Spain) under Grant PB 93-81 and by INTAS under Grant 94-929.

\section{References}

Christov, C. I. \& Velarde, M. G. [1995] "Dissipative solitons," Physica D86, 323-347.

Chua, L. O. [1993] "Global unfolding of Chua's circuits," IEICE Trans. Fundamental Electron. Commun. Comput. Sci. E76A5, 704-737.

Huang, G. \& Velarde, M. G. [1996] "Head-on collisions of dark solitons near the zero-dispersion point in optical fibers," Phys. Rev. E54, 3048-3051.

Hasegawa, A. \& Kodama, Y. [1995] Solitons in Optical Communications (Oxford University Press, Oxford).

Kuramoto, Y. [1984] Chemical Oscillators, Waves and Turbulence (Springer-Verlag, Berlin).

Madan, R. N. (Ed.) [1993] Chua's Circuit: A Paradigm for Chaos (World Scientific, Singapore).

Murray, J. D. [1993] Mathematical Biology (SpringerVerlag, Berlin).

Nekorkin, V. I. \& Chua, L. O. [1993] "Spatial disorder and wave fronts in a chain of coupled Chua's circuits," Int. J. Bifurcation and Chaos 3, 1282-1292.

Nekorkin, V. I. \& Velarde, M. G. [1994] "Solitary wave, soliton bound states and chaos in a dissipative Korteweg-de Vries equation," Int. J. Bifurcation and Chaos 4, 1135-1146.

Nekorkin, V. I., Kazantsev, V. B., Rulkov, N. F., Velarde, M. G. \& Chua, L. O. [1995] "Homoclinic orbits and solitary waves in a one-dimensional array of Chua's circuits," IEEE Trans. Circuits Syst. 42, 785-801.

Nekorkin, V. I., Kazantsev, V. B. \& Velarde, M. G. [1996] "Travelling waves in a circular array of Chua's circuits," Int. J. Bifurcation and Chaos 6, 473-484.

Nepomnyashchy, A. A. \& Velarde, M. G. [1994] "A threedimensional description of solitary waves and their interaction in Marangoni-Benard layers," Phys. Fluids 6, 187-198.

Ogorzalek, M. J., Galias, Z., Dabrowski, A. M. \& Dabrowski, W. R. [1995] "Chaotic waves and spatiotemporal patterns in large arrays of doubly-coupled Chua's circuits," IEEE Trans. Circuits Syst. 42, 706-715.

Perez Marino, I., de Castro, M., Perez-Munuzuri, V., Gomez-Gesteira, M., Chua, L. O. \& Perez-Villar, V. [1995] "Study of Reentry Initiation in Coupled Parallel Fibers," IEEE Trans. Circuits Syst. 42, 665-672.

Perez-Munuzuri, A., Perez-Munuzuri, V., Perez-Villar, V. \& Chua, L. O. [1993] "Spiral waves on a 2-D array of nonlinear circuits," IEEE Trans. Circuits Syst. 40, 872-877.

Perez-Munuzuri, V., Perez-Villar, V. \& Chua, L. O. [1993] "Travelling wave front and its failure in onedimensional array of Chua's circuits," J. Circuits Syst. Comput. 3, 215-229. 
Saarloos, W. \& Hohenberg, P. C. [1992] "Fronts, pulses, sources and sinks in generalized complex GinzburgLandau equation," Physica D56, 303-367.

Shashkov, M. V. \& Turaev, D. V. [1996] "On the complex bifurcation set for a system with simple dynamics," Int. J. Bifurcation and Chaos 6, 949-968.

Shilnikov, L. P. [1969] "On a new type of bifurcation of multidimensional dynamical systems," Sov. Math. Dokl. 10, 1368-1371.

Shilnikov, L. P. [1970] "A contribution to the problem of the structure of an extended neighborhood of a rough equilibrium state of saddle-focus type," Math. USSR Sb. 10, 91-100.
Turaev, D. V. [1996] "On dimension of non-local bifurcational problems," Int. J. Bifurcation and Chaos 6 , 919-948.

Velarde, M. G., Nekorkin, V. I. \& Maksimov, A. G. [1995] "Further results on the evolution of solitary waves and their bound states of a dissipative Korteweg-de Vries equation," Int. J. Bifurcation and Chaos 5, 831-839.

Winfree, A. T. [1991] "Varieties of spiral wave behavior: An experimantalist's approach to the theory of excitable media," Chaos 1, 303-334.

Zhabotinsky, A. M. [1974] Concentration Autooscillations (Nauka, Moscow) (Russian). 